\title{
Moderate Algorithm for Generalized Artificial Neural Network
}

\author{
B.M.Singhal \\ Department of Computer Application, ITM - UNIVERSE, GWALIOR, \\ India.
}

\begin{abstract}
In the process of learning we may presume the neural networks are simplified models of the biological neurons system. The Artificial Neural Network ( ANN ) is an information processing system which is inspired by brain learning system. It is assumed that brain is composed of a large number of highly interconnected processing elements working in groups to solve specific problems. Various networks and algorithms have been proposed to enhance the machine learning process and to achieve some thing new. In this paper we have proposed a moderate algorithm for most generalized multilevel artificial neural network, which may be reduced to various other forms of neural networks.
\end{abstract}

Key Words- Algorithm, Neural Networks

\section{INTRODUCTION}

Artificial Neural Network is a precise tool to represent a technology that is in the disciplines of neurosciences, mathematics, physics, computer science and engineering. The advent of neural networks saw the area of artificial intelligence grows rapidly as a tool for many undecided events. These studies have huge possibilities to design a competent Artificial Neural Network resembling and enhancing brain efficiencies. To achieve the goal many techniques and algorithm in this field have been proposed by various authors. Here we propose a moderate algorithm for most generalized multilevel artificial neural network, which may be reduced to various other forms of neural networks.

Let $x_{11}, \ldots, x_{1 r_{1}}$ is a set of inputs of first stage of generalized ANN and the corresponding weights are $w_{11}, \ldots, w_{1 r_{1}}$. Then the strength of $x_{1 k}$ will be

$$
w_{1 k} x_{1 k} ; \mathrm{k}=1,2, \ldots, r_{1}
$$

And let we have $m_{1 k}$ neurons of type $x_{1 k}$. Then the combined probabilistic strength of type ${ }^{x_{1 k}}$ will be

$$
\prod_{k=1}^{r_{1}} \frac{\left(w_{1 k} x_{1 k}\right)^{m_{1 k}}}{m_{1 k} !}
$$

If we choose an activation function

$$
f_{1}\left(\sum_{k=1}^{r_{1}} m_{1 k}\right)
$$

the system output for first stage of generalized ANN with the weight vector

$$
\mathrm{W}=\left\{w_{11}, w_{12}, \ldots, w_{1 r_{1}}\right\}
$$

may be taken as $\left(0 \leq w_{1 i} \leq 1\right)$

$$
f_{1}\left(\sum_{k=1}^{r_{1}} m_{1 k}\right) \prod_{k=1}^{r_{1}} \frac{\left(w_{1 k} x_{1 k}\right)^{m_{1 k}}}{m_{1 k} !}
$$

Similarly for other stages of generalized ANN, we have the outputs respectively as

$$
\begin{gathered}
f_{2}\left(\sum_{k=1}^{r_{2}} m_{2 k}\right) \prod_{k=1}^{r_{2}} \frac{\left(w_{2 k} x_{2 k}\right)^{m_{2 k}}}{m_{2 k} !}, \ldots \\
\quad f_{n}\left(\sum_{k=1}^{r_{s}} m_{n k}\right) \prod_{k=1}^{r_{s}} \frac{\left(w_{n k} x_{n k}\right)^{m_{n k}}}{m_{n k} !}
\end{gathered}
$$

Finally, the total output $\mathrm{Z}$ with an unified activation function

$$
f\left(\sum_{k=1}^{r_{1}} m_{1 k}+\ldots+\sum_{k=1}^{r_{s}} m_{n k}\right)
$$

may be taken as below (Because in case of indefinite large numbers the probabilistic models are best suited for infinity )

$$
\begin{aligned}
& \left.\sum_{\left(m_{1 r}\right)=0}^{\infty} \ldots \sum_{\left(m_{n s}\right)=0}^{\infty}{ }^{r_{1}} m_{1 k}+\ldots+\sum_{k=1}^{r_{s}} m_{n k}\right) \\
& f\left(\sum_{k=1}^{r_{1}}\left(\sum_{k=1}^{r_{1}} m_{1 k}\right) \quad f_{n}\left(\sum_{k=1}^{r_{s}} m_{n k}\right)\right. \\
& \prod_{k=1}^{r_{1}} \frac{\left(w_{1 k} x_{1 k}\right)^{m_{1 k}}}{m_{1 k} !} \ldots \prod_{k=1}^{r_{s}} \frac{\left(w_{n k} x_{n k}\right)^{m_{n k}}}{m_{n k} !} \\
& \sum_{m_{1}, m_{2}, \ldots, m_{n}=0}^{\infty} f\left(m_{1}+m_{2}+\ldots+m_{n}\right) \\
& \prod_{i=1}^{n} f_{i}\left(m_{i}\right) \frac{\left(\sum_{j=1}^{r_{1}} w_{1 j} x_{1 j}\right)^{m_{1}}}{m_{1} !} \\
& \frac{\left(\sum_{j=1}^{r_{s}} w_{n j} x_{n j}\right)^{m_{n}}}{m_{n} !} \ldots \ldots \ldots
\end{aligned}
$$


by using the Identity [9].

$$
\begin{aligned}
& \sum_{m_{1}, m_{2}, \ldots, m_{n}=0}^{\infty} f\left(m_{1}+m_{2}+\ldots+m_{n}\right) \prod_{i=1}^{n} f_{i}\left(m_{i}\right) \\
& \frac{\left(\sum_{j=1}^{r_{1}} x_{1 j}\right)^{m_{1}}}{m_{1} !} \frac{\left(\sum_{j=1}^{r_{s}} x_{n j}\right)^{m_{n}}}{m_{n} !} \\
& \sum_{\left(m_{1 r}\right)=0}^{\infty} \cdots \sum_{\left(m_{n s}\right)=0}^{\infty} \\
& f\left(\sum_{k=1}^{r_{1}} m_{1 k}+\ldots+\sum_{k=1}^{r_{s}} m_{n k}\right) \\
& f_{1}\left(\sum_{k=1}^{r_{1}} m_{1 k}\right) \quad f_{n}\left(\sum_{k=1}^{r_{s}} m_{n k}\right) \\
& \prod_{k=1}^{r_{1}} \frac{\left(x_{1 k}\right)^{m_{1 k}}}{m_{1 k} !} \prod_{k=1}^{r_{s}} \frac{\left(x_{n k}\right)^{m_{n k}}}{m_{n k} !} \\
& f\left(\sum_{i=1}^{n} m_{i}\right)
\end{aligned}
$$

In case of symmetrical layers

in R.H.S. of (1), may be taken as constant say, $\delta$. Also a group of neurons are similar in nature so $f_{i}\left(m_{i}\right)$ may be taken as modular parameters $\lambda_{i}^{m_{i}}$, say. So we have

$$
\begin{gathered}
\sum_{\mathrm{Z}=\delta}^{\infty} \sum_{m_{1}, m_{2}, \ldots, m_{n}=0} \frac{\left(\lambda_{1} \sum_{j=1}^{r_{1}} w_{1 j} x_{1 j}\right)^{m_{1}}}{m_{1} !} \ldots \\
\frac{\left(\lambda_{n} \sum_{j=1}^{r_{s}} w_{n j} x_{n j}\right)^{m_{n}}}{m_{n} !} \\
\left.\lambda_{1} \sum_{j=1}^{r_{1}} w_{1 j} x_{1 j}+\ldots+\lambda_{n} \sum_{j=1}^{r_{s}} w_{n j} x_{n j}\right)
\end{gathered}
$$

$$
\left.\lambda_{\operatorname{Exp}(} \sum_{j=1}^{r_{1}} w_{1 j} x_{1 j}+\ldots+\lambda_{n} \sum_{j=1}^{r_{s}} w_{n j} x_{n j}\right)
$$

That is, in generalized artificial neural network, the out put is directly proportional to exponentially weighted sum of inputs and middle stages outcomes along with constraints

$$
\lambda_{1}, \ldots, \lambda_{n} \text {; which may be suitably defined as per network }
$$

Also if the network configures with

$$
\lambda_{i}=1 ; \mathrm{i}=1,2, \ldots, \mathrm{n}
$$

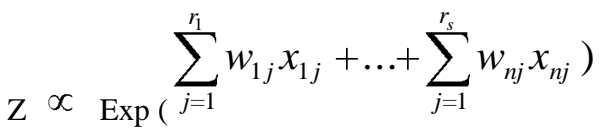

$$
\begin{aligned}
& \propto_{\operatorname{Exp}}\left(W_{1} X_{1}+\ldots+W_{n} X_{n}\right) \\
& \text { Where } W_{i} X_{i}=\sum_{j=1}^{r_{i}} w_{i j} x_{i j}
\end{aligned}
$$

This is a generalization of the author's result [10], i.e.

$$
\mathrm{Z} \propto \operatorname{Exp}(\mathrm{WX})
$$

\section{CONCLUSION}

In this paper we have proposed a moderate algorithm for most generalized artificial neural network, further work on application is being carried out and some fruitful results are expected in near future.

\section{REFERENCES}

[1]. J.Moody and C. Darken, "Fast learning in Networks of locally- tuned Processing units, Neural Computation , 1:281294, 1989.

[2]. N.B.Karayiannis and G.W. Mi, "Growing radial basis Neural Networks: merging supervised and unsupervised learning with network growth techniques " IEEE Trans. On Neural Networks.

[3]. D.Dasgupta and S. Forest, "Artificial Immune System in Industrial Applications” Proc. Of the IPMM'99, 1999.

[4]. P.Hajela and J.S.Yoo, "Immune Network Modeling in design Optimization ".In new Ideas in Optimization,(Eds) D Corne, M.Dorigo \& F. Glover, McGraw Hill, London, pp. 203-215, 1999.

[5]. L.N.De Castro and F.J.Von Zuben, "An Evolutionary Immune Network for data clustering ", Proc. Of the IEEE Brazelian Symposium on Neural Networks, pp. 84-89, 2000 b.

[6]. D.S.Broomhead and D.Lowe, "Multivariate functional Interpolation and adaptive Networks", Complex Systems, 2:321-355, 1988 .

[7]. M.J.D. Powell, "Radial Basis Functions for multivariable Interpolation", A reviw in IMA Conference, Algorithm for Appr. Of Functions and Data, J.C. Mason \& M.G. Cox (eds.), Oxford , U.K.: Oxford Univ. Press, 143-167, 1987.

[8]. C.A. Michelli, "Interpolation of Scattered Data: Distance Matrices and conditionally Positive definite Functions", Const.Approx.,2: 11-22, 1986.

[9]. B.M. Singhal and B.M. Agrawal, "On Multiple Integrals Involving Hypergeometric Functions of two Variables", Jnanabha Sect. A. Vol. 4, July 1974.

[10]. B.M. Singhal, "A proposed Algorithm for Multivariate Artificial Neural Network", accepted for publication, IEEE Conference March.2010 Indian Institute Of Science, Banglore, India. http://www.ijcaonline.org/ archives/number3/8183 